\title{
Functional dissection of $\gamma$-aminobutyric acid metabolism in Neurospora crassa
}

\author{
Kamila Ďurišová1, Martin Šimkovič ${ }^{1}$, L’udovít Varečka ${ }^{1}$, Michal Kaliňák ${ }^{2}$, Katarína Šoltys ${ }^{3,4,5}$, \\ Barbora Mosná ${ }^{1}$ and Svetlana Kryštofová ${ }^{1}$ \\ ${ }^{1}$ Institute of Biochemistry and Microbiology, Faculty of Chemical and Food Technology, Slovak University of Technology in \\ Bratislava, Bratislava, Slovakia \\ ${ }^{2}$ Central Laboratories, Faculty of Chemical and Food Technology, Slovak University of Technology in Bratislava, Bratislava, \\ Slovakia \\ ${ }^{3}$ Department of Molecular Biology, Faculty of Natural Sciences, Comenius University in Bratislava, Bratislava, Slovakia \\ ${ }^{4}$ Department of Microbiology and Virology, Faculty of Natural Sciences, Comenius University in Bratislava, Bratislava, \\ Slovakia \\ ${ }^{5}$ Comenius University Science Park, Comenius University in Bratislava, Bratislava, Slovakia
}

\begin{abstract}
The GABA shunt is one of the metabolic pathways that is ubiquitous in prokaryotes and eukaryotes. $\gamma$-aminobutyric acid (GABA) in fungi is required in the stress responses, virulence and development. The number of genes encoding glutamate decarboxylase (gad), GABA transaminase ( $g t a)$ and succinic semialdehyde dehydrogenase (ssadh) varies between fungal species. The genome-wide analysis in Neurospora crassa resulted in the identification of a gta and a ssadh. Disruption of either gta or ssadh decreased respiration rate and biomass accumulation, reduced growth on GABA and beta-alanine. The gta and ssadh mutants exhibited aberrant hyphal morphology and displayed differential transcription of the GABA shunt genes. In the gta mutant, protoperithecia and perithecia formation was almost completely suppressed in the presence of GABA and beta-alanine, indicating GTA requirement for the turnover of these amino acids. The strains displayed differential metabolic dysregulations in response to different nitrogen sources. The phenotypic differences between the gta and ssadh mutants could be contributed to accumulation of intermediates of the GABA shunt and/or GABA shunt-independent functions. Together, our data suggest that the GABA shunt could function as a moderate modulator of multiple biological events, including respiration, energy metabolism, carbon and nitrogen metabolism, growth, as well as sexual development in $N$. crassa.
\end{abstract}

Key words: GABA - GABA transaminase - Succinic semialdehyde dehydrogenase - Fungi Neurospora crassa

Abbreviations: GABA, $\gamma$-aminobutyric acid; CMF, crude mitochondrial fraction; DON, deoxynivalenol; GAD, glutamate decarboxylase; GTA, GABA transaminase; NAD, nicotinamide adenine dinucleotide; NCCR, NADH cytochrome c reductase; ROS, reactive oxygen species; SCM, synthetic crossing medium; SSADH, succinic semialdehyde dehydrogenase; VM, Vogel's minimal agar medium.

Correspondence to: Svetlana Kryštofová, Institute of Biochemistry and Microbiology, Faculty of Chemical and Food Technology, Slovak University of Technology in Bratislava, Radlinského 9, 81237 Bratislava, Slovakia

E-mail: svetlana.krystofova@stuba.sk

Kamila Ďurišová, Institute of Biochemistry and Microbiology, Faculty of Chemical and Food Technology, Slovak University of Technology in Bratislava, Radlinského 9, 81237 Bratislava, Slovakia

E-mail: kamila.durisova@stuba.sk 


\section{Introduction}

$\gamma$-aminobutyric acid (GABA) is a ubiquitous non-proteinogenic amino acid that is synthesized from glutamate by glutamate decarboxylase (GAD) in the cytosol. It is then transported to mitochondria and converted by GABA transaminase (GTA) and succinic semialdehyde dehydrogenase (SSADH) to succinic acid. All three enzymes form GABA shunt, a metabolic cycle that is attached to the tricarboxylic acid cycle (TCA) (Kumar and Punekar 1997; Fait et al. 2008). The last reaction in the GABA shunt also generates $\mathrm{NADH}$. Succinate and NADH can serve as substrates for oxidative phosphorylation. GABA is not just a metabolite that enters mitochondria to boost the energy metabolism, but it fulfils other biological functions. In bacteria, it confers resistance to acidic $\mathrm{pH}$ and controls quorum sensing (Chevrot et al. 2006; Feehily and Karatzas 2013). Mammals use GABA as the primary inhibitory neurotransmitter in the brain (Hampe et al. 2017; Ravasz et al. 2017). The plants and fungi require GABA in the response to stress conditions (Cao et al. 2013; Mead et al. 2013; Bönnighausen et al. 2015; Priya et al. 2019; Seifikalhor et al. 2019). Although in mammals, GABA binds to GABA receptors and inhibits neuron response, in fungi, GABA receptors have not been identified yet. Our previous study indicated GABA regulatory function in conidia germination and hyphal development (Nižnanský et al. 2013). In plants, GABA regulates aluminium-activated malate transporters, and these proteins subsequently function as modulators of plant growth and development (Ramesh et al. 2018).

The physiological role of GABA in the fungal growth and development remains still elusive. The GABA shunt presence in Neurospora crassa was first confirmed by Schmit and Brody (1975) and Christensen and Schmit (1980). A decade later, glutamate decarboxylase was isolated from N. crassa, and its activity determined in early stages of germination and conidiation (Hao and Schmit 1991, 1993). No more reports have been since then published about the GABA shunt function or regulation in this fungus. In contrast to $N$. crassa, the GABA shunt has been thoroughly investigated in yeasts where it is involved in the reactive oxygen species (ROS) resistance, osmotolerance, heat-shock tolerance, longevity and polyhydroxybutyrate synthesis (Bach et al. 2009; Cao et al. 2013). In the filamentous fungus Aspergillus nidulans, the acidic $\mathrm{pH}$, carbon and nitrogen catabolite repression, and hypoxia regulate GABA permease expression (Hutchings et al. 1999; Peñalva et al. 2008; Masuo et al. 2010). In the mycoparasitic fungus Trichoderma atroviride, the gad expression was upregulated in submerged cultures as well as after illumination (Pokorný et al. 2005). The GAD activity in T. atroviride was differentially regulated during development (Strigáčová et al. 2001), and its deletion caused complete inhibition of GAD, moderate decline in biomass accumulation, significant reduction in respiration and lower thermotolerance (Nižnanský et al. 2013, 2016).

The most recent reports indicate that the fully functional GABA shunt plays an important role in fungal pathogenesis. Deletion of two GABA transaminases in Fusarium graminearum led to reduced virulence and mycotoxin (deoxynivalenol, DON) production due to heightened sensitivity to oxidative stress, increased levels of GABA and inability to utilize putrescine (Bönnighausen et al. 2015). The disruption of GABA metabolism by deletion of one ssadh (out of the predicted three homologs) in Stagonospora nodorum only slightly decreased the virulence (Mead et al. 2013). In Pyricularia oryzae, the deletion of one gta and one ssadh rendered strains displaying reduced pathogenicity (Guo et al. 2011).

To this day, a comprehensive study demonstrating a more complex view on the GABA shunt in filamentous fungi has not been published yet. In the previous studies, usually only single genes with the same enzymatic functions have been explored. To our knowledge, this is the first study in which the entire catabolic branch of the GABA shunt is being investigated. As a model organism, we used the non-pathogenic filamentous fungus N. crassa, in which the genome-wide search identified a single gta and ssadh. Our primary goal was to evaluate how these enzymes contribute to the mitochondrial function, energy metabolism, carbon and nitrogen metabolism as well as development in response to nutritional cues.

\section{Materials and Methods}

\section{Strains and culture conditions}

Wild type strains of Neurospora crassa, ORS-SL6a (FGSC 4200; mat $a$ ) and 74-OR23-1VA (FGSC 2489; mat A), and knock-out strains, $\Delta$ gta (FGSC 20481; gta::hph mat a) and $\Delta$ ssadh (FGSC 15637; ssadh::hph mat a) were obtained from the Fungal Genetics Stock Center, Kansas State University, USA (McCluskey 2003; McCluskey et al. 2010). The strains were maintained on Vogel's minimal agar medium (VM; Vogel 1956, 1964) that contained 2\% sucrose and 1.5\% agar. Submerged cultures were grown in liquid VM for $16 \mathrm{~h}$ at $28^{\circ} \mathrm{C}$ and $200 \mathrm{rpm}$ or in liquid synthetic crossing medium (SCM) for $24 \mathrm{~h}$ at $28^{\circ} \mathrm{C}$ and $200 \mathrm{rpm} .8$-day-old conidia were used as inoculum at the final concentration of $1 \times 10^{6} \mathrm{ml}^{-1}$.

SCM was used for the vegetative growth and the sexual crosses (Westergaard and Mitchell 1947). The conidial suspension $(1 \mu \mathrm{l})$ was inoculated in the center of the SCM or the modified SCM. The SCM contained $10 \mathrm{mM} \mathrm{KNO}_{3}$ as a sole nitrogen source and $1.5 \%$ sucrose as a carbon source. The modified SCM was composed of $5 \mathrm{mM} \mathrm{KNO}_{3}$ and $5 \mathrm{mM}$ of an alternative nitrogen source $(\gamma$-aminobutyric acid, $\beta$-alanine or putrescine). To determine changes in 
colony morphology, strains were cultured on solid medium at $28^{\circ} \mathrm{C}$ in continuous light for 6 days and photographed by Canon SX160 IS (Canon, Japan). Protoperithecia formation was induced by nitrogen starvation on the SCM or modified SCM. Strains grew on the SCM for 6 days at $25^{\circ} \mathrm{C}$ in the continuous light. Protoperithecia were fertilized with conidia of the wild type of the opposite mating type on the sixth day of cultivation. Perithecia morphology and ascospore ejection were observed after 10-14 days following fertilization. The morphology of hyphae, protoperithecia and perithecia was determined by compound microscopy using Axio Imager A1 (Carl Zeiss, Germany).

\section{Determination of respiration in submerged cultures}

The wild type and $\Delta g t a$ and $\Delta s s a d h$ strains were grown in liquid VM with or without GABA $(5 \mathrm{mM})$ at $28^{\circ} \mathrm{C}$ and $200 \mathrm{rpm}$. The amounts of consumed $\mathrm{O}_{2}$ and released $\mathrm{CO}_{2}$ were measured by OPTIMA 7 Combustion Analyzer (MRU, Germany) at approximately $2 \mathrm{~h}$ intervals. The biomass accumulation was determined as dry weight at the same time points. The growth rate was calculated from biomass accumulation in the exponential phase of growth. It was calculated using the linear regression and defined as biomass dry weight per an hour per volume unit. The cultivation lasted for $32 \mathrm{~h}$. The analysis of each strain was carried out in three independent experiments and expressed as a mean value with standard deviation.

\section{$R N A$ isolation and $R T-q P C R$}

For RNA isolation, mycelium from submerged cultures was harvested by vacuum filtration, rinsed with water and frozen in liquid nitrogen. Frozen mycelium was crushed with a pestle in a precooled mortar filled with liquid nitrogen. RNA was isolated from disintegrated mycelium using TRI Reagent (Merck, Germany), and cDNA was synthesized from the total RNA using MMLV reverse transcriptase (Life Technologies, USA), dNTP's (Biotechrabbit, Germany) and oligo $\mathrm{dT}_{15}$ (Promega, USA) according to manufacturer's protocols. The qPCR was carried out in Stratagene Mx3005P system (Agilent Technologies, USA) using cDNA as a template, gene specific primers (Table 1) (Metabion, Germany) and 5x HOT FirePol EvaGreen HRM Mix (no ROX) (Solis BioDyne, Estonia) according to manufacturers' protocols. The gta, ssadh and hh3 (histone 3, NCU01635) amplicons were 162, 113 and 133 bp in length. qPCR conditions were: initial denaturation at $95^{\circ} \mathrm{C}$ for $12 \mathrm{~min}$ followed by 40 cycles of denaturation at $95^{\circ} \mathrm{C}$ for $15 \mathrm{~s}$, annealing at $56-61^{\circ} \mathrm{C}$ for $20 \mathrm{~s}$ and elongation at $72^{\circ} \mathrm{C}$ for $20 \mathrm{~s}$. To verify the specificity of the amplified fragment, a melting curve was determined afterward. All measurements were carried out with three technical replicates in two independent experiments. The $h h 3$ was used as the internal reference for calculating the normalized differences in threshold cycles $(\mathrm{Ct})$. To normalize the expression level of a gene of interest (GOI) to a housekeeping gene (HKG), the expression levels of the two genes were divided according to the equation from the manufacturer's manual: $\mathrm{Ef}^{-\mathrm{Ct}(\mathrm{GOI})} / \mathrm{Ef}^{\mathrm{Ct}(\mathrm{HKG})}=\mathrm{Ef}^{-[\mathrm{Ct}(\mathrm{GOI})-\mathrm{Ct}(\mathrm{HKG})]}=\mathrm{Ef}^{-\Delta \mathrm{Ct}}$.

\section{NMR spectroscopy}

The strains were grown in liquid SCM $\left(24 \mathrm{~h}, 28^{\circ} \mathrm{C}, 200 \mathrm{rpm}\right.$, in the dark) or in liquid VM $\left(16 \mathrm{~h}, 28^{\circ} \mathrm{C}, 200 \mathrm{rpm}\right.$, in the dark). The mycelium was collected and processed as described previously in Nižnanský et al. (2013). Dry mycelium was extracted in $50 \%$ methanol at $4^{\circ} \mathrm{C}$ for $8 \mathrm{~h}$. The final

Table 1. List of primers used in this study

\begin{tabular}{|c|c|c|c|c|}
\hline Gene & Primer & Sequence $\left(5^{\prime}-3^{\prime}\right)$ & Source & Amplicon size (bp) \\
\hline \multirow{2}{*}{$h h 3$} & hH3-F & GCGTCTCGTCCGTGAGATTG & \multirow{2}{*}{ RT-qPCR } & \multirow{2}{*}{133} \\
\hline & $\mathrm{hH} 3-\mathrm{R}$ & TTAGCGTGGATAGCGCAGAG & & \\
\hline \multirow{2}{*}{ gta } & GTA-F & CTTTGCTCTTCAAGGCCATC & \multirow{2}{*}{ RT-qPCR } & \multirow{2}{*}{162} \\
\hline & GTA-R & CAATGAACGTTCCCTGTCCT & & \\
\hline \multirow{2}{*}{ ssadh } & SSADH-F & AGGTGGTCAAGCTGGCTAAC & \multirow{2}{*}{ RT-qPCR } & \multirow{2}{*}{113} \\
\hline & SSADH-R & ACCAACCATGCCAACCTCAA & & \\
\hline \multirow{2}{*}{ gad-1 } & GAD1-F & CATCGCACAACCATCCTAAA & \multirow{2}{*}{ RT-qPCR } & \multirow{2}{*}{148} \\
\hline & GAD1-R & ACACCCTTACTCTGGCGTTG & & \\
\hline \multirow{2}{*}{ gad-2 } & GAD2-F & GCAGTGGATCATTCCCAACT & \multirow{2}{*}{ RT-qPCR } & \multirow{2}{*}{133} \\
\hline & GAD2-R & CTCCGTAACCGACACCAAGT & & \\
\hline \multirow{2}{*}{$\Delta g t a$} & NCGTA-F & CCAGGAACTAAGTCAGTCCAACGAC & \multirow{2}{*}{ 5'flank, PCR } & \multirow{2}{*}{1300} \\
\hline & HPH-R & GAGGGCAAAGGAATAGAGTAGATGC & & \\
\hline \multirow{2}{*}{$\Delta s s a d h$} & NCSSADH-F & TCCTCCGACAAGATCTTGTGAAACG & \multirow{2}{*}{ 5'flank, PCR } & \multirow{2}{*}{1400} \\
\hline & HPH-R & GAGGGCAAAGGAATAGAGTAGATGC & & \\
\hline
\end{tabular}


extract was concentrated in vacuum concentrator (Eppendorf, Germany) and solubilized in deuterated water (Merck, Germany) containing sodium salt of trimethylsilylpropionic acid-2,2,3,3-d4 (TSP) (Merck, Germany) and 0.02\% $\mathrm{NaN}_{3}$ to prevent bacterial contamination. Spectra were measured in $5 \mathrm{~mm}$ NMR tubes on a Varian/Agilent VNMRS NMR spectrometer operating at $599.75 \mathrm{MHz}$ using tnnoesy pulse sequence with water pre-saturation during relaxation (1 s), $100 \mathrm{~ms}$ mixing time and $4 \mathrm{~s}$ acquisition time (Saude et al. 2006) accumulating 128 scans. The spectra were analysed and concentrations determined in Chenomx NMR Suite software v8.3.

\section{NCCR assay}

The crude mitochondrial fraction (CMF) was isolated according to Šimkovič et al. (2001) from the mycelium cultured in liquid $\mathrm{VM}$ for $16 \mathrm{~h}$ at $28^{\circ} \mathrm{C}$ and $200 \mathrm{rpm}$. Mycelium was collected by filtration and disintegrated by grinding with a pestle in an ice-cold mortar that contained homogenization buffer and glass beads. The homogenization lasted $1 \mathrm{~min}$ and the sample was then rested on the ice for $1 \mathrm{~min}$. The mycelium disintegration was completed after 15 repeats of homogenization. The homogenization buffer was composed of $500 \mathrm{mM}$ sorbitol, $50 \mathrm{mM}$ Tris/ $\mathrm{HCl} \mathrm{pH}$ 7.4, $1 \mathrm{mM}$ PMSF (phenylmethanesulfonyl fluoride), $1 \mathrm{mM}$ ATP and $1 \mathrm{mM}$ EDTA (ethylenediaminetetraacetic acid). The mitochondrial membrane fraction was isolated by differential centrifugation. The mycelial debris and glass beads were discarded after the first centrifugation in Avanti J30-I (Beckman Coulter, USA $)$ at $2,500 \times g\left(4^{\circ} \mathrm{C}\right.$ and $\left.10 \mathrm{~min}\right)$. The $\mathrm{CMF}$ was isolated after the second centrifugation at $10,000 \times g\left(4^{\circ} \mathrm{C}\right.$ and $30 \mathrm{~min}$ ). The membrane pellet was afterward resuspended in the homogenization buffer. The protein concentration was quantified by the Bradford assay (Bradford 1976). The NADH cytochrome c reductase (NCCR) activity was determined by reduction of $20 \mu \mathrm{M}$ cytochrome $\mathrm{c}$ according to Tisdale (1967). One unit of the activity was defined as the amount of the enzyme that reduced $1 \mu \mathrm{mol}$ of oxidized cytochrome $c$ in one second $\left(\varepsilon_{550} 21,100 \mathrm{M}^{-1} \cdot \mathrm{cm}^{-1}\right)$. The NCCR specific activity was expressed in $\mu \mathrm{mol} / \mathrm{s}$ per $\mu \mathrm{g}$ proteins. Three independent experiments were performed. The mean values with standard deviations were calculated from three technical replicates.

\section{Results}

\section{Identification of GABA shunt genes in Neurospora crassa and their expressions}

To identify the homologs of GAD, GTA and SSADH in N. crassa genome database, GAD from T. atroviride, $\mathrm{Gta}_{1} /$
$\mathrm{Gta}_{2}$ from F. graminearum and UGA2 from Sacharomyces cerevisiae (Goffeau et al. 1996; Nižnanský et al. 2013; Bönnighausen et al. 2015) were used as queries in the NCBI BLAST searches. We identified two putative gad genes, gad-1 (NCU06803) and gad-2 (NCU00678), one gta (NCU08998) and one ssadh (NCU00936). GAD-1 and GAD-2 shared 69\% and $47 \%$ identity with GAD from $T$. atroviride, respectively. GTA and SSADH from N. crassa had 56 and 70\% identity with the homologs from $F$. graminearum and S. cerevisiae, respectively. The $\Delta g t a$ (FGSC 20481) and the $\Delta s s a d h$ (FGSC 15637) strains were obtained from the Fungal Genetic Stock Centre (USA). The homologous gene replacement with the hygromycin resistance gene $(h p h)$ was verified by PCR (data not shown). The reverse primers annealed to the $h p h$ sequence while forward primers were complementary to the region located upstream of the 5 'flank of the cassette. The amplicon sizes were $1300 \mathrm{bp}$ for the gta locus and $1400 \mathrm{bp}$ for the ssadh locus (Table 1). In addition, disruption of gta and the ssadh genes were confirmed by lack of the gta and the ssadh transcripts (Fig. 1), as well as by enzymatic assays (data not shown).

Transcriptional profiles of $N$. crassa GABA shunt genes were analyzed in strains grown in submerged medium containing either nitrate (SCM) or ammonium salts (VM). The relative expressions of gad-1, gad-2, gta and ssadh were determined by the RT-qPCR (Fig. 1). The histon 3 gene, $h h 3$, was used as the internal reference. The $\mathrm{gad}-1$ and $\mathrm{gad}-2$ displayed different expression profiles. Deletion of the gta or the ssadh caused dramatic changes in the gad-2 expression. The gad-2 expression levels increased in both mutants. Contrary to the gad-2, the gad-1 transcript remained unaffected. In $\Delta g t a$, the gad-2 increased 11-fold in VM and 35-fold in SCM cultures. Deletion of the gta also led to ssadh upregulation. The increase was 3-fold in VM and 11-fold in SCM. In contrast to ssadh overexpression in $\Delta g t a$ strain, the gta levels in $\Delta s s a d h$ strain changed moderately. The gta expression rose 2 -fold in VM and declined 2-fold in SCM. The effect of gad-1 and gad-2 mutations on GABA shunt gene expressions is absent from this study, since it would require rather extensive study that covers isolation of single and double mutants, establishing redundancy between $\mathrm{gad}-1$ and gad-2 as well as epistatic analysis between both gad genes and upstream regulators.

\section{Deletion of gta and ssadh in N. crassa affects hyphal develop- ment, colony morphology and sexual development}

The deletion of gta and the ssadh did not influence macroscopic appearance of mutants cultured on solid VM. The phenotypic aberrations were however observed on microscopic levels in submerged cultures (Fig. 2). When compared to the wild type, the $\Delta g t a$ strain formed thicker leading hyphae with enlarged vacuoles. The branched hyphae were thicker at the base and very thin at the tips. In 

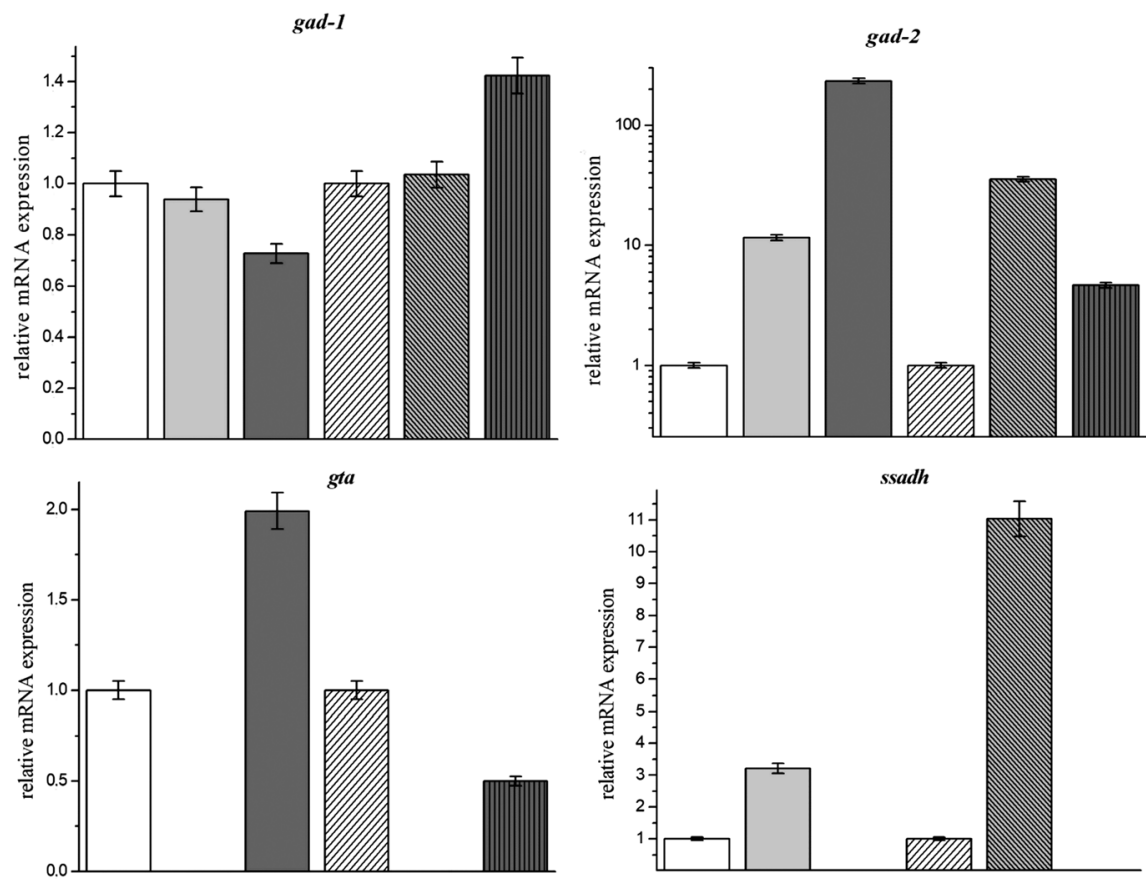

Figure 1. Relative expression of gad-1, gad-2, gta and ssadh in the N. crassa strains in liquid VM and SCM. The relative gene expressions in $\Delta g t a$ and $\Delta s s a d h$ were normalized with the $h h 3$ (histone 3) expression and compared with the expressions in the wild type (WT). Means of three sets of experiments with standard deviations are shown.

contrast to the $\Delta g t a$ strain, hyphae of the the $\Delta s s a d h$ were shorter, thinner and fragile than in the wild type. In addition, extremely short "dwarf-like" branch hyphae covered the hyphal surface of the $\Delta s s a d h$ strain hyphae indicating defects in hyphal branching.

We also elucidated the ability of the $\Delta g t a$ and $\Delta s s a d h$ strains to utilize different nitrogen sources. Previous studies demonstrated that some filamentous fungi can transport and metabolize GABA as a nitrogen source (Nižnanský et al. 2013, 2016; Bönnighausen et al. 2015) while others lack this ability (Mead et al. 2013). For this reason, we surveyed the ability of $N$. crassa to utilize GABA and other nitrogen sources, such as putrescine and $\beta$-alanine that require GABA shunt enzymes in the turnover. The results revealed that $N$. crassa was not able to grow on GABA as a sole nitrogen source. In subsequent experiments, the strains were cultured on SCM supplemented with GABA or other nitrogen sources. When compared with SCM containing a sole nitrate, $5 \mathrm{mM}$ putrescine had effect neither on colony growth nor aerial hyphae differentiation and conidiation (Fig. 3A). In contrast to putrescine, $5 \mathrm{mM} \mathrm{GABA}$ and $\beta$-alanine inhibited growth, aerial mycelium formation and conidiation. The defects were more pronounced in the $\Delta g t a$ and the $\Delta s s a d h$ strains than in the wild type.

In this study, we observed influence of GABA, $\beta$-alanine and putrescine on protoperithecia and perithecia formation
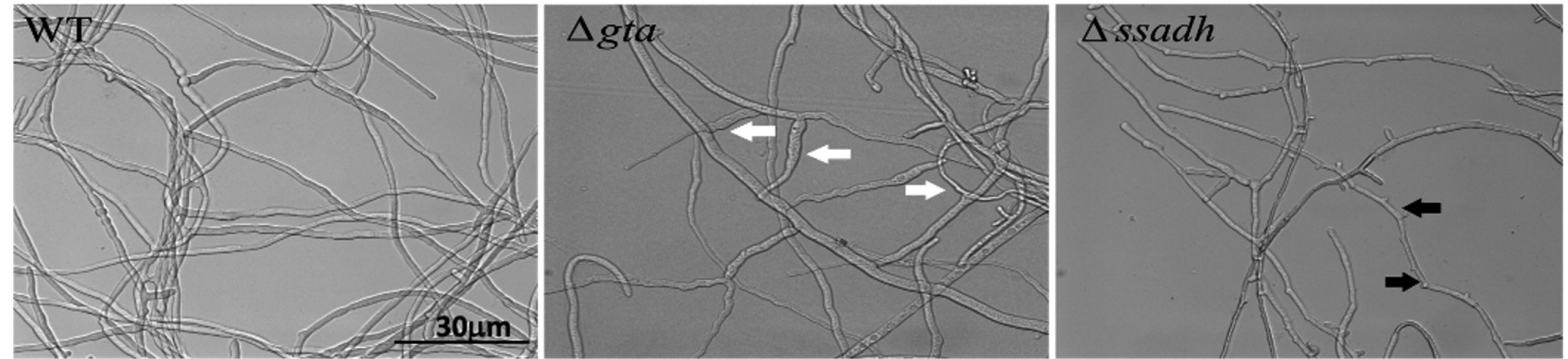

Figure 2. Hyphal morphology of wild type (WT), $\Delta g t a$ and $\Delta s s a d h$ grown in liquid VM. $\Delta g t a$ formed hyphae of uneven thickness with much wider diameter at the base of branches and very thin at the hyphal tips (white arrows). Hyphae in $\Delta s s a d h$ were much thinner and fragile than WT and their surface was covered by dwarf-like branches (black arrows). The bar in the images represents $30 \mu \mathrm{m}$ in length. 
(Fig. 3B). Putrescine, GABA and $\beta$-alanine demonstrated moderate inhibitory effect on formation of protoperithecia and development of perithecia in the wild type and the $\Delta s s a d h$ strain. However, deletion of gta in $\Delta g t a$ strain caused nearly complete inhibition of protoperithecia and perithecia formation in presence of $5 \mathrm{mM} \mathrm{GABA}$ and $\beta$-alanine indicating involvement of gta in the sexual development of N. crassa grown in presence of GABA or $\beta$-alanine (Fig. 3B). In the $\Delta g t a$ strain, the morphology of protoperithecia and perithe-

A

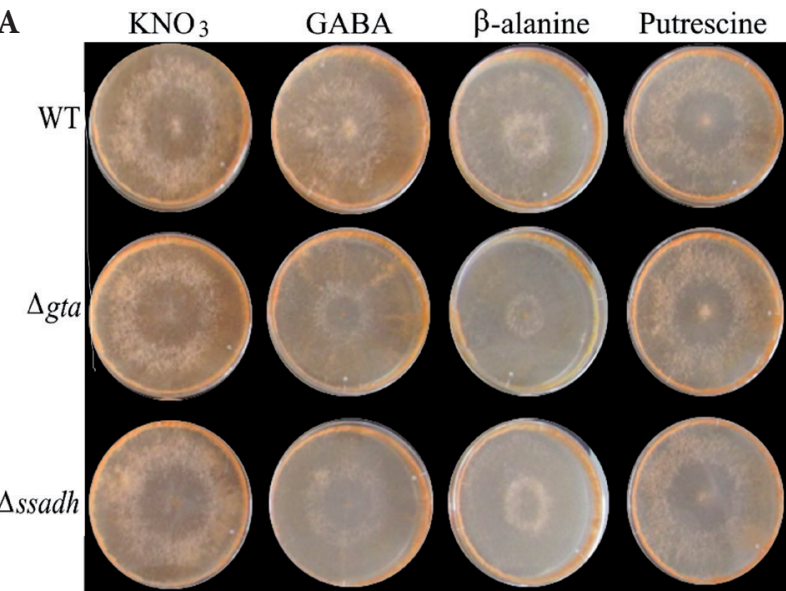

B

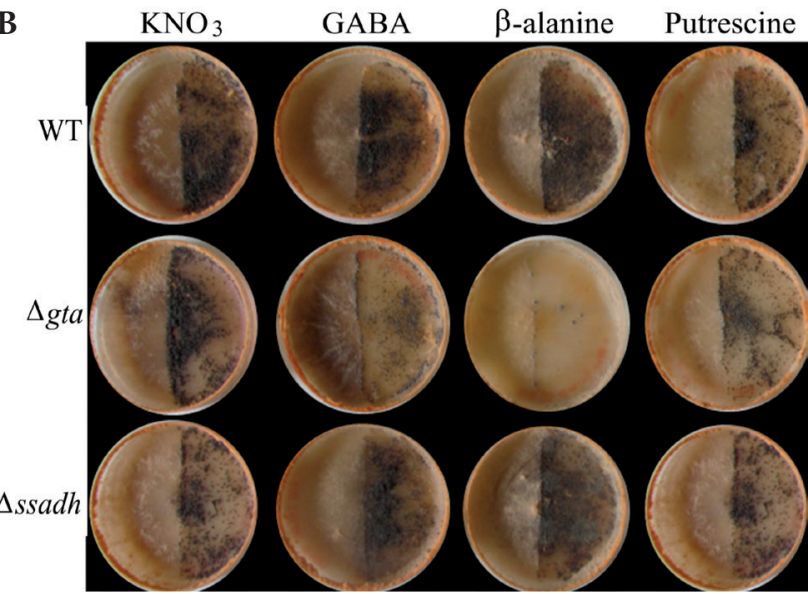

Figure 3. A. Colony morphology of strains grown on SCM supplemented with GABA, $\beta$-alanine and putrescine for 6 days in the light at $28^{\circ} \mathrm{C}$. GABA and $\beta$-alanine supplementation reduced hyphae formation and conidiation in mutant strains. Negative effect caused by $\beta$-alanine was more pronounced in $\Delta g t a$ and $\Delta s s a d h$. Putrescine did not show any effect on mutant asexual development. B. Perithecia formation by the wild type (WT), $\Delta g t a$ and $\Delta s s a d h$ (mat a) on SCM supplemented with GABA, $\beta$-alanine and putrescine 14 days after fertilization of 6-day old protoperithecia with FGSC 2489 ( mat A). Protoperithecia and perithecia were formed at $25^{\circ} \mathrm{C}$ in continuous light. Perithecia development was suppressed by GABA and $\beta$-alanine in $\Delta g t a$ whereas other strains remained unaffected. Right half of the plate culture was fertilized by macroconidia of FGSC 2489 cia was adversely affected (Fig. 4A,B). Protoperithecia were embedded in the agar, and perithecia were larger with short or missing beaks (beak is a narrow, upper part of perithecium with opening in the centre required for ascospore ejection).

\section{Growth and respiration rate are reduced in $\Delta g t a$ and $\Delta$ ssadh strains}

To better understand the relationship between GABA metabolism and mitochondrial respiration, we measured growth rates, $\mathrm{O}_{2}$ consumption and $\mathrm{CO}_{2}$ production in submerged cultures of $N$. crassa grown in liquid VM in the presence of GABA ( $5 \mathrm{mM}$ ) or the absence of GABA (Fig. $5 A)$. The growth rate of the wild type was maximal in the exponential growth phase between 6 and $18 \mathrm{~h}$ of cultivation and reached $0.24 \pm 0.02 \mathrm{mg}$ dry weight $/ \mathrm{h} / \mathrm{ml}$. Both mutants, $\Delta g t a$ and $\Delta s s a d h$, grew at slower growth rates than the wild type: $\Delta g t a$ at $0.18 \pm 0.03$ and $\Delta s s a d h$ at $0.16 \pm 0.03$. Although, GABA presence in the culture medium did not affect the growth rates of the strains in the exponential phase dramatically, it had a significant impact on biomass accumulation during the stationary phase between 18 and $32 \mathrm{~h}$. While the dry weight of the wild type in VM without GABA reached $3.2 \mathrm{mg} / \mathrm{ml}$ in the stationary phase, in the GABA enriched $\mathrm{VM}$ it was $4.3 \mathrm{mg} / \mathrm{ml}$. The highest biomass concentrations determined in $\Delta g t a$ and $\Delta s s a d h$ strains grown without GABA were very similar, 2.7 and $2.8 \mathrm{mg} / \mathrm{ml}$. Both strains reached these concentrations at $18 \mathrm{~h}$ of cultivation, then they gradually decreased down to $0.9 \mathrm{mg} / \mathrm{ml}$ at $32 \mathrm{~h}$. As shown in Fig. 5A, the GABA supplementation slowed down the biomass decline in $\Delta g t a$ and $\Delta s s a d h$ strains indicating as-yet-unknown GABA biological function in the stationary growth phase.

At the same time, determination of $\mathrm{O}_{2}$ consumption and $\mathrm{CO}_{2}$ formation confirmed a correlation between the respiration rate and biomass accumulation (Fig. 5A). During cultivation of the wild type in VM medium, the highest $\mathrm{O}_{2}$ loss (expressed as $\mu \mathrm{mol}\left(\mathrm{O}_{2}\right) / \mathrm{mg}$ dry weight or $\mathrm{n} \%$ ) was observed in the exponential phase of fungal growth (up to $18 \mathrm{~h}$ ). In the stationary phase, the amount of $\mathrm{O}_{2}$ did not change. The amount of released $\mathrm{CO}_{2}$ in the wild type mirrored the amount of consumed oxygen and was as high as $18 \%$ in the stationary phase. The $\Delta g t a$ strain consumed a similar amount of $\mathrm{O}_{2}$ as the wild type but $\mathrm{CO}_{2}$ production in the mutant was significantly slower. The impact of ssadh mutation on respiration was comparable to that observed in the $\Delta g t a$ strain. The decline in respiration of both defective strains, although not as drastic, confirmed that both genes contribute to the energy metabolism in $N$. crassa.

GABA catabolism catalysed by GTA and SSADH generates one molecule of NADH and succinate. Both can be used as electron donors by the electron transport chain. To determine respiration rate in mutants, NADH 
A

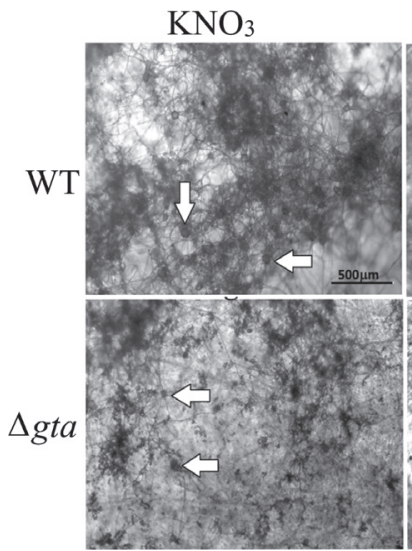

GABA

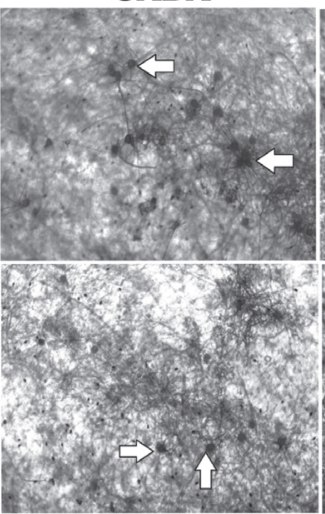

$\beta$-alanine

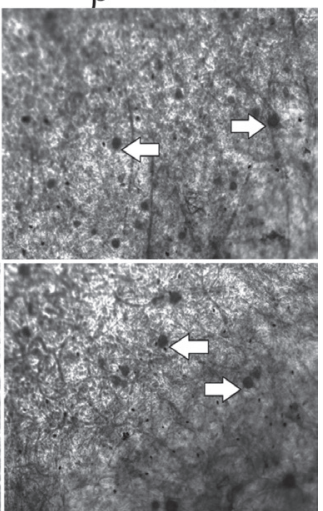

B

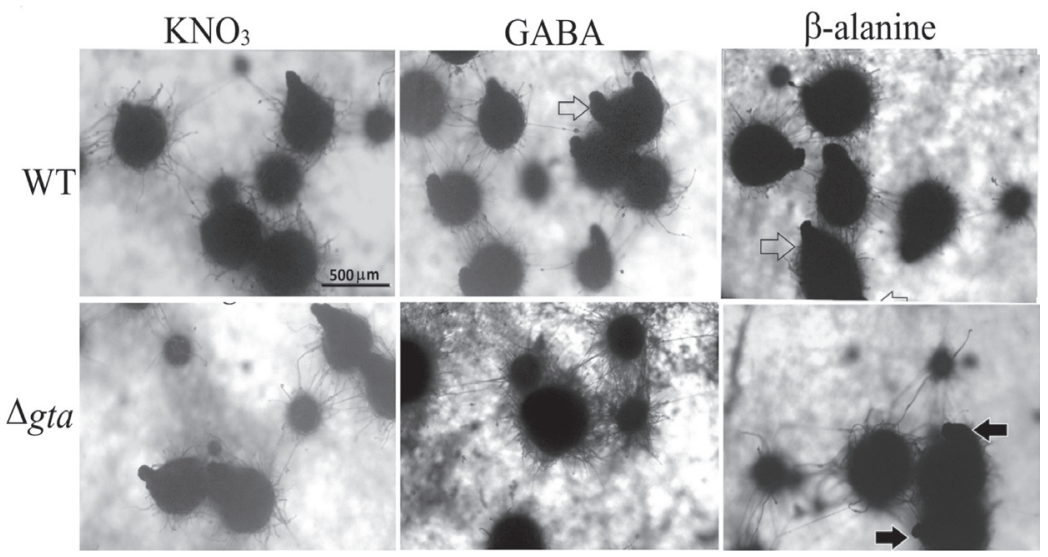

Figure 4. A. Microscopy of protoperithecia in WT and $\Delta g t a$. White arrows point to protoperithecia. The bar is $500 \mu \mathrm{m}$ in length. B. Microscopy of perithecia in WT and $\Delta g t a$. GABA and $\beta$-alanine increased formation of two-beak perithecia in WT (unfilled arrows), reduced perithecia number and led to development of immature and larger perithecia with no or short beaks in $\Delta g t a$ (black arrows). The bar is $500 \mu \mathrm{m}$ in length. cytochrome $\mathrm{c}$ reductase assay was performed. We isolated crude mitochondrial fraction (CMF) from submerged cultures and determined NADH cytochrome $\mathrm{c}$ reductase activity in CMF (Fig. 5B). In comparison to the wild type, NADH cytochrome c reductase activity appeared higher in the $\Delta g t a$ strain, although the difference between the two strains was of low statistical significance $(p>0.05)$. Contrary to the $\Delta g t a$ strain, the $\Delta s s a d h$ strain had NADH cytochrome c reductase activity reduced by $50 \%$ when compared to the wild type. The significant difference was determined at $p<0.05$ (Fig. 5B).

\section{GABA shunt disruptions are associated with metabolomic changes}

To identify dysregulated metabolites in GABA shunt mutants, the metabolomic profile was elucidated by NMR of 28 intracellular metabolites (Fig. 6A,B). Strains were cultured in two media with different nitrogen sources: $\mathrm{NH}_{4} \mathrm{NO}_{3}(\mathrm{VM})$ and $\mathrm{KNO}_{3}(\mathrm{SCM})$. The metabolites that we expected having altered levels would have been either substrates or products of the GABA shunt. Surprisingly, no GABA accumulation was observed in the mutants. The GABA concentration did not significantly changed in the $\Delta g t a$ strain while the $\Delta s s a d h$ strain had more than $40 \%$ reduction in GABA during nitrogen limitation (SCM) (Fig. 6B). The $\Delta s s a d h$ also exhibited $30 \%$ decrease in succinate concentration. Succinate remained unaffected in the $\Delta g t a$ and $\Delta s s a d h$ strains grown in VM. Glutamine and glutamate remained unaltered in both mutants grown in VM (Fig. 6A). However, they increased 20-30\% when both mutants were cultured in SCM. Although, no dramatic changes in the GABA shunt metabolism were determined in mutants, the strain lacking ssadh exhibited more alterations than $\Delta g t a$ strain.

Metabolic profiles (Fig. 6A,B) in the mutants changed in response to two different nitrogen sources. We determined statistically significant changes in amino acid and carbohydrate levels in both mutants grown either in the VM or SCM. While in VM cultures only 12 out of 28 metabolites displayed significant differences, the concentrations changed drastically (Fig. 6A). In contrast, 20 metabolites exhibited significantly altered concentrations in the SCM, but the changes were moderate (Fig. 6B). The $\Delta g t a$ strain, when grown in VM, increased concentrations of several amino acids (Fig. 6A). Aspartate and asparagine rose 40 and 6.7fold, isoleucine 30-fold. Differences in alanine, arginine and lysine were statistically insignificant. Changes in glycine, 


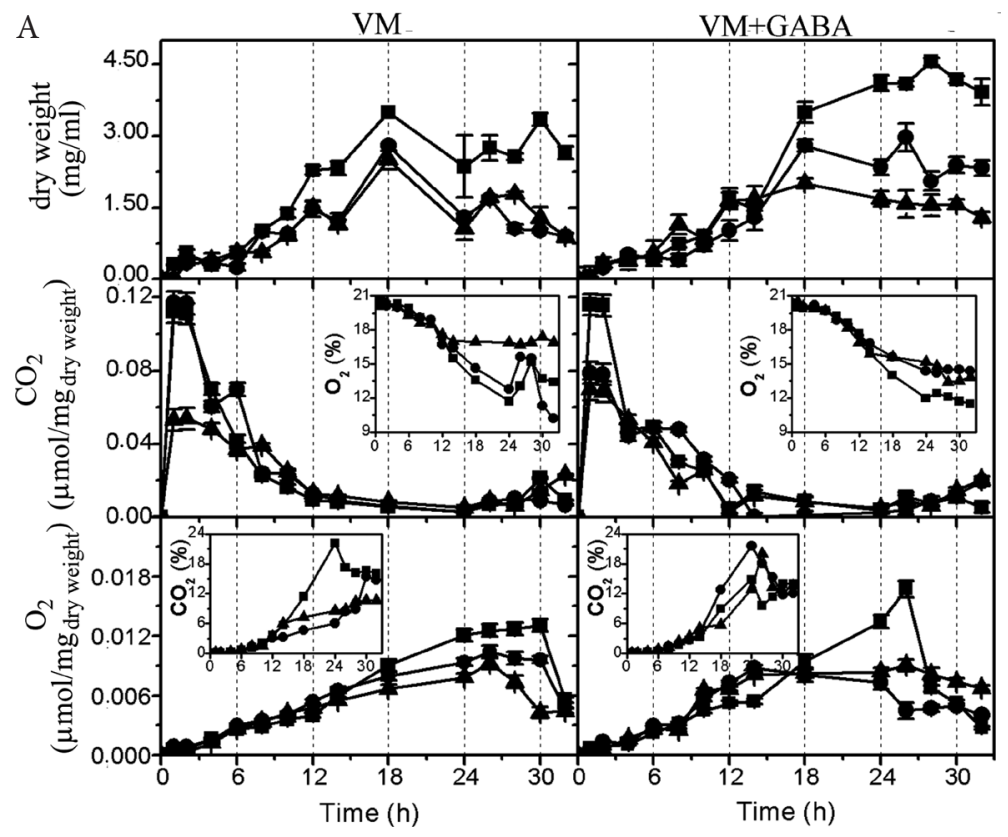

$\mathrm{B}$

Figure 5. A. Growth and respiration in submerged culture. Strains were grown in VM (the left part of the figure) and in GABA supplemented medium (the right part of the figure) at $28^{\circ} \mathrm{C} . \mathrm{O}_{2}$ consumption and $\mathrm{CO}_{2}$ release are expressed as gas weight per biomass dry weight. The internal graphs show $\mathrm{O}_{2}$ or $\mathrm{CO}_{2}$ percentage by volume. Wild type (WT)-squares, $\Delta$ gta-circles, $\Delta s s a d h$-triangles. B. The specific activity of NADH cytochrome c reductase in $N$. crassa strains. The strains were cultured in liquid VM at $28^{\circ} \mathrm{C}$ for $16 \mathrm{~h}$. In $\mathrm{A}$ and $\mathrm{B}$, representative of three experiments is shown. Means with standard deviations were calculated from triplicates.

leucine and threonine remained also unaltered. The $\Delta s s a d h$ strain differed from $\Delta$ gta strain in that glycine concentration decreased 14.5-fold. While the glycine level significantly dropped, aspartate and asparagine rose up to 14.6-fold and asparagine 1.8-fold, respectively. Significant deviations from the wild type were also determined in carbohydrates (Fig. 6A). Glucose decreased 2-fold in $\Delta g t a$ and 5-fold in $\Delta s s a d h$. While trehalose and glycerol displayed minimal alterations in mutant strains, mannitol increased in $\Delta s s a d h$ 5.5-times. As expected, significant changes were determined in some components of the TCA cycle and glycolysis. Lactate was reduced in $\Delta g t a 3.3$-fold and $\Delta s s a d h 7$-fold; fumarate rose 6-fold in $\Delta g t a$ and 9-fold in $\Delta s s a d h$. The precursors of phospholipid metabolism, ethanolamine and choline were dysregulated in the $\Delta g t a$, whereas they remained unchanged in the $\Delta s s a d h$ (Fig. 6A).

The conidiophore formation can be observed in the $N$. crassa cultured in SCM. The differentiation triggers development of different cell types that are not present in the submerged mycelium from the nitrogen-rich VM. We therefore expected that the metabolomic profiles in the VM and SCM cultures would vary from one another. Surprisingly, the differences were not found in amino acid profiles but in carbohydrates, organic acids and phospholipid precursors. The concentrations of amino acids in the mutants grown in VM or SCM were either unaffected or elevated (Fig. 6A,B).
Contrary to the VM cultures, no significant changes were determined in carbohydrates, choline and ethanolamine in the $\Delta g t a$ strain cultured in SCM. The $\Delta s s a d h$ displayed intracellular accumulation of glucose (5-fold), 3-fold reduction of trehalose and 40\% decrease in mannitol (Fig. 6B). Organic acids from the TCA cycle, succinate, fumarate and malate coordinately declined in both mutants, indicating downregulation of respiration activity due to lower GABA flux passing through the GABA shunt and TCA. Furthermore, the decrease in TCA cycle rate was accompanied by 2 -fold increase in lactate generated by glycolysis. The metabolomic analysis of the $\Delta g t a$ and $\Delta s s a d h$ strains illustrated a complexity of metabolic changes triggered by dysregulation in GABA shunt.

\section{Discussion}

A recent study demonstrated that $N$. crassa lives, regardless what has been thought before, interesting lifestyle, switching between three states: saprotrophic, endophytic and pathogenic (Kuo et al. 2014). In plant-fungus interactions, $N$. crassa had to evolve various strategies in dealings with the plant defence system. When exposed to environmental stress, plants increase synthesis of several components, such as polyamines, ROS, phytohormones and amino acids 

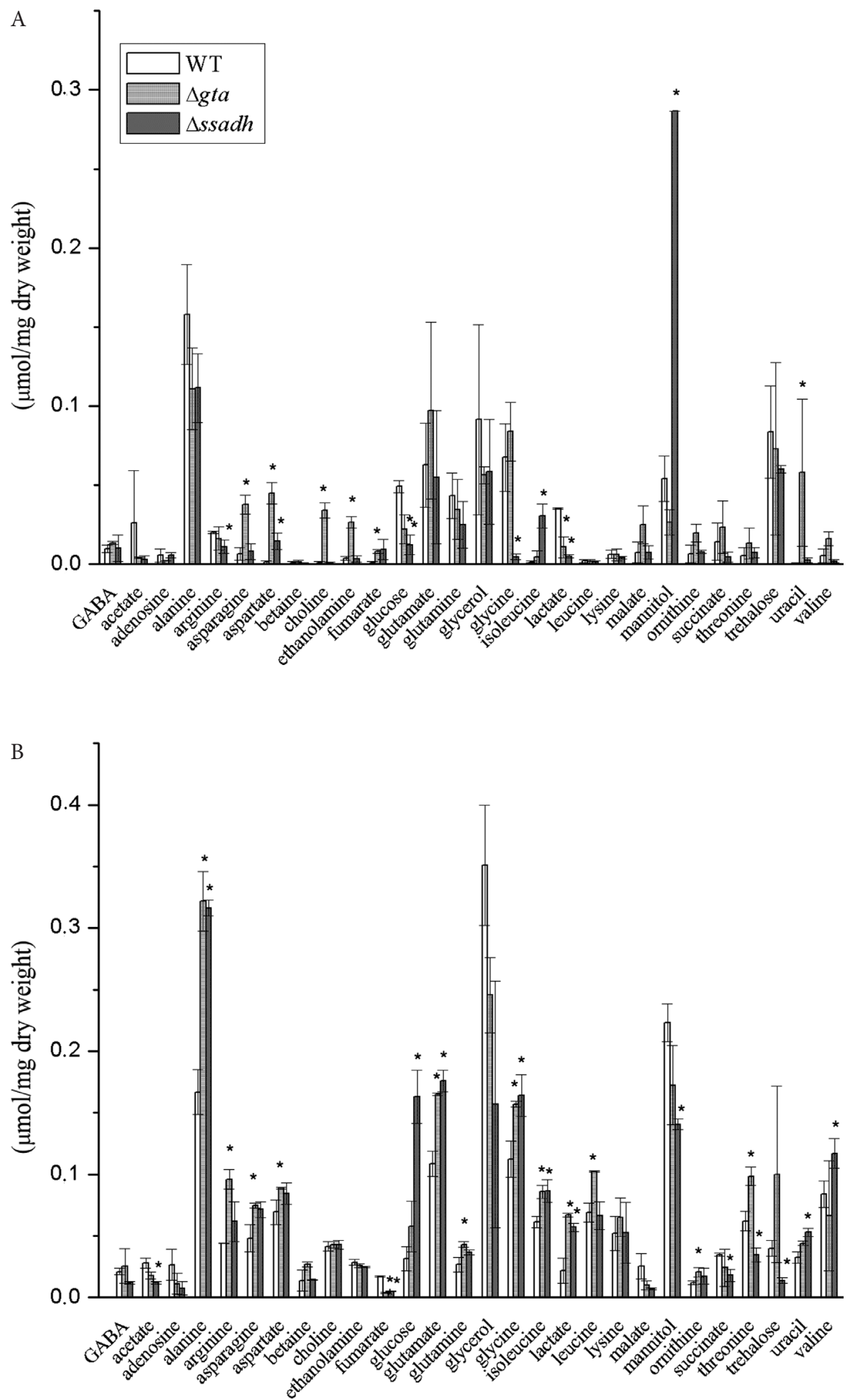

Figure 6. NMR metabolomics of N. crassa strains grown in liquid medium with different nitrogen sources. A. Strains were cultured in $\mathrm{VM}\left(\mathrm{NH}_{4} \mathrm{NO}_{3}\right)$ for $16 \mathrm{~h}$ at $28^{\circ} \mathrm{C}$. B. Strains were cultured in $\mathrm{SCM}\left(\mathrm{KNO}_{3}\right)$ for $24 \mathrm{~h}$ at $28^{\circ} \mathrm{C}$. Representative of three experiments is shown. Statistical significance was determined by calculating two-tailed $p<0.05$. The asterisks indicate statistically significant differences. 


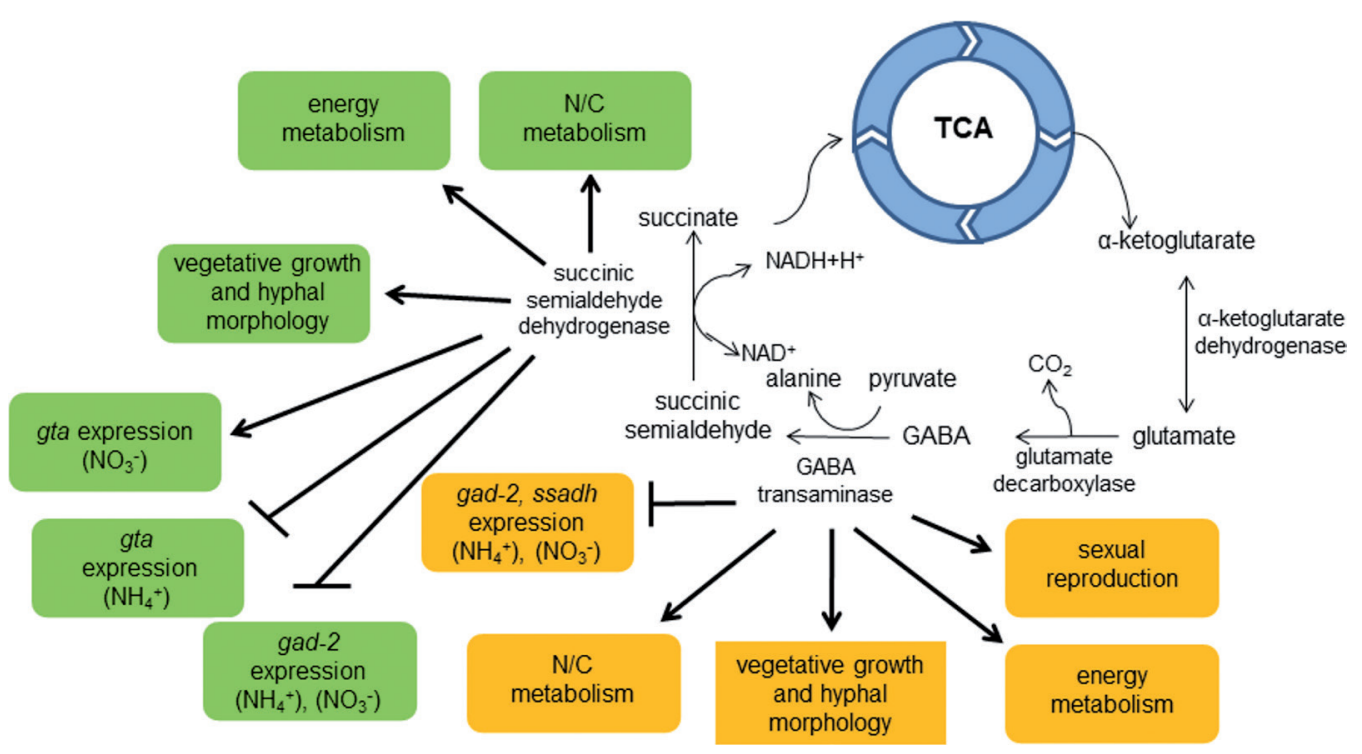

Figure 7. Model of functional dissection of GABA metabolism in N. crassa. The GABA shunt genes gta and ssadh modulate multiple fungal processes: utilization of nitrogen sources, carbon and nitrogen $(\mathrm{N} / \mathrm{C})$ metabolism, asexual growth, respiration and energy metabolism. Disruptions in the GABA shunt resulted in upregulation of $\mathrm{gad}-2$, ssadh and variable gta expression.

(Bouché et al. 2003; Shelp et al. 2012). In plants, GABA produced during pathogen attacks can be then utilized by fungal phytopathogens to replenish their own TCA cycle. Strains deficient in GTA and SSADH activity were not able to grow on GABA as a sole nitrogen source. Deletion of the GABA catabolic genes gta or ssadh in fungi led to reduction of F. graminearum, S. nodorum and P. oryzae virulence, indicating that the fully functional GABA shunt is important in their pathogenesis (Guo et al. 2011; Mead et al. 2013; Bönnighausen et al. 2015).

The agar plate assays in this study revealed that $N$. crassa was not able to utilize the extracellular GABA as the sole nitrogen source (data not shown). This finding was very similar to that reported in the wheat pathogen $S$. nodorum (Mead et al. 2013). This was however in contrast to F. graminearum and T. atroviride that were able to grow on GABA as the sole nitrogen source (Nižnanský et al. 2013; Bönnighausen et al. 2015). The genome analysis in this study revealed that the $N$. crassa genome is missing the GabA homolog that encodes a hypothetical GABA permease (Hutchings et al. 1999), giving a possible explanation to growth impairment on agar medium with only GABA as the nitrogen source. We assume that GABA could be transported into mycelium by non-specific means, likely using other amino acid permease(s). This suggests that the GABA uptake itself is not a prerequisite for the pathogenesis, but the fully functional GABA shunt is.

Fungi and plants synthesize polyamines (Shelp et al. 2012; Chen et al. 2019). The most common polyamines in fungi are putrescine and spermidine; less common is spermine.
Polyamines are essential for fungal growth and differentiation (Valdés-Santiago et al. 2012). Their depletion results in growth restriction whereas high intracellular accumulation of polyamines may be toxic. GABA is a major intermediate of polyamine degradations in eukaryotes including fungi. Deletion of both gta in F. graminearum significantly reduced fungal growth, inferring both transaminases as crucial enzymes of putrescine catabolism (Bönnighausen et al. 2015). In N. crassa, it was demonstrated that it is not able to transport extracellular polyamines, and the fungus tolerates high levels of putrescine (Barnett et al. 1988; Davis and Ristow 1989). Deletion of gta and ssadh in N. crassa therefore did not affect growth and sexual development on culture medium supplemented by putrescine (Fig. $3 \mathrm{~A}, \mathrm{~B}$ ). In contrast to putrescine, both $\Delta g t a$ and $\Delta s s a d h$ displayed reduction in aerial hyphae formation and conidiation on GABA and $\beta$-alanine. This could be explained by the structural similarity between GABA and $\beta$-alanine. Consequently, we could suggest that $\beta$-alanine is a substrate of $N$. crassa GTA. Human GABA transaminase exhibits similar affinity toward GABA and $\beta$-alanine and converts them to succinic semialdehyde and malonate semialdehyde (Tiedje et al. 2010). F. graminearum $\Delta \Delta g a_{1,2}$ strains exhibited partial substrate specificity toward GABA and $\beta$-alanine. Gta ${ }_{1}$ showed preference to $\beta$-alanine whereas $\mathrm{Gta}_{2}$ to GABA (Bönnighausen et al. 2015). Fungal strains deficient in GTA and SSADH activity were not able to grow on GABA as sole nitrogen source. Deletion of the GABA catabolic genes gta or ssadh in fungi led to reduction of F. graminearum, S. nodorum and P. oryzae virulence, indicating that the fully functional GABA shunt is important 
in their pathogenesis (Guo et al. 2011; Mead 2013; Bönnighausen et al. 2015).

Nitrogen starvation has long been known to stimulate development of female sexual structures, protoperithecia that increase in size after fertilization, and form flask-like perithecia with asci and ascospores inside (Nelson and Metzenberg 1992). In this study, we have firstly demonstrated the differential effect of $g t a$ and $s s a d h$ mutations on asexual and sexual development. The gta deletion almost completely reduced sexual development in the presence of $\beta$-alanine and GABA whereas $\triangle$ ssadh remained unaffected (Fig. 3B). This perhaps could explain the inability of the fungal strains defective in gta to form sexual reproductive structures when grown on culture medium that could eventually contain traces of GABA or $\beta$-alanine. Based on our data (not shown) even $0.1 \mathrm{mM}$ significantly reduces the number of perithecia. The inhibitory action of GABA or $\beta$-alanine on sexual development could not be simply explained by the intracellular increase of these amino acids. One possible interpretation of this phenomenon lies in that the fungal GABA and $\beta$-alanine might be involved in signalling or regulation of other proteins in protoperithecial development. GTA would therefore counteract GABA effect showing positive impact on protoperithecia formation (Fig. 7). In our previous work on T. atroviride (Nižnanský et al. 2013), we demonstrated 2 -fold decrease in chloride transport in $\mathrm{gad}$ mutants which indicated unspecified changes in membrane transport of the strain lacking GABA. In plant root tips, GABA interacts with anion transporters that belong to the family of aluminiumactivated malate transporters and can transport GABA (Ramesh et al. 2018). These transporters strongly regulate intracellular GABA levels in response to acidic and alkaline $\mathrm{pH}$. In our study with gta and ssadh mutants, the concentration of GABA remained at low levels like in the wild type. The association of GABA with membrane transporting system, similar to that in plants, would provide a plausible explanation for maintaing intracellular GABA in $N$. crassa mutants.

Nitrogen (SCM) or carbon starvation induced differentation in N. crassa submerged cultures, which is demonstrated by conidiophore formation (Guignard and Brody 1983; Guignard et al. 1984; Müller and Russo 1989; Ebbole 1998). The $\Delta g t a$ and $\Delta s s a d h$ strains also differed in hyphal morphology (Fig. 2). The morphology of the mutants seems to be contradictory. $\Delta g t a$ formed thick hyphae while $\Delta s s a d h$ hyphae were thin and fragile. The difference could be contributed to accumulation of the intermediates, GABA and SSA. High intracellular SSA levels are believed to be toxic. Consequently, the toxic metabolite accumulates in mitochondria and leads to decreased NCCR specific activity in $\Delta s s a d h$ (Fig. 5B). The specific activity of the NCCR in $\Delta g t a$ however remained unaltered in comparison to the wild type. When the strains were stained by Mito Tracker CMXROS (data not shown), the fluorescence signal in $\Delta s s a d h$ was lower than in $\Delta g t a$ indicating reduced number of mitochondria in $\Delta s s a d h$. In a previous study, deletion of $g t a_{1}$ and $g t a_{2}$ in $F$. graminearum reduced mitochondrial respiration in presence of GABA (Bönnighausen et al. 2015). Decrease in respiration was also observed in a T. atroviride strain deficient in GABA synthesis (Nižnanský et al. 2013). Notwithstanding differences, both strains showed lower respiratory activities as well as slower biomass accumulation at the early stages of growth, suggesting that GABA shunt might be required for germination of conidia. Perhaps, it is not coincidental that this is also the stage where high activity of GAD was determined in N. crassa (Christensen and Schmit 1980). The high GAD activity in germlings could be explained by a sudden demand to charge energy metabolism in the cells transitioning from a dormant to vegetative state. Another stage, where the demand for energy increases is conidiation. The high GAD activity was determined in conidia of N. crassa and T. atroviride (Strigáčová et al. 2001; Pokorný et al. 2005). The late stationary phase in N. crassa was affected by mycelium deterioration in $\Delta g t a$ and $\Delta s s a d h$ (Fig. $5 A)$. The medium supplementation by GABA slowed down the biomass decrease in both mutants. In aging or starving mycelium, GABA might therefore play a protective role and/ or function as a storage component. This correlates with the previous study in T. atroviride where GAD activity rose dramatically in stationary phase (Strigáčová et al. 2001).

The deletion of individual genes of GABA catabolism also affects the nitrogen and carbon metabolism of N. crassa, which was demonstrated by NMR spectroscopy (Fig. 6A,B). The metabolic shifts between mutants and the wild type in some metabolites were significant. This could be contributed to an adaptation process. $\Delta g t a$ deletion mutants in F. graminearum exhibited high accumulation of GABA in mycelium (Bönnighausen et al. 2015). We had expected a similar effect in $N$. crassa. However, our NMR results rendered rather low GABA concentrations in both GABA shunt mutants. Furthermore, the GABA levels did not change significantly in comparison to the wild type. This likely indicated adjustments in the associated metabolic pathways such as TCA cycle or amino acid metabolism. However, the changes were rather moderate but cumulative. In both mutants, amino acid pool demonstrated shifts toward increased levels while intermediates of TCA cycle decreased. To dissect this phenomenon correctly, future studies would have to implement alternative approaches such as more detailed metabolomics and study of metabolic fluxes.

Although we did not directly determine changes in ROS formation in $\Delta g t a$ and $\Delta s s a d h$ strains, their increase in $\Delta s s a d h$ strain was indicated by dramatic rise in mannitol. The metabolite is known to function as a ROS scavenger in fungi. Fungi use mannitol as a storage sugar, which is produced in response to stress conditions such as oxidative stress or high temperatures. It also plays a role in metabolism and 
pathogenesis. In S. nodorum, it was required for vegetative sporulation (Meena et al. 2015). In fungi, GABA shunt has been implicated in protection against excessive intracellular concentration of ROS. Strains deficient in GTA and SSADH were sensitive to oxidative stress (Cao et al. 2013; Mead et al. 2013; Bönnighausen et al. 2015).

In conclusion, we would like to stress several interesting aspects of fungal GABA shunt that have been inferred by our study (Fig. 7). They are as follows: a) high plasticity in response to stress, b) moderating function of central carbon and nitrogen metabolisms, c) modulating function in mitochondrial activity and d) co-regulating function of fungal development. We believe that better understanding of this overlooked pathway could contribute to dissection of interspecies interactions between fungi and other organisms as well as development of novel antifungal agents.

Author's contribution. The authors, S. K., K. D. and M. Š. designed the experiments and wrote the manuscript. The experimental work was preformed by K. D., M. Š. and B. M. RT-qPCR and NMR analyses were carried out by K. $\check{D}$. in collaboration with K. $\check{S}$., M. K. and L. V.

Acknowledgment. The project was supported by the Slovak Research and Development Agency under the contracts no. APVV-16-0216 and APVV-16-0439 and by Biotechnology Research in the Collaboration with Academic Institutions No. ITMS 26220220093.

Conflict of interest. The authors declare that they have no conflict of interest.

\section{References}

Bach B, Meudec E, Lepoutre JP, Rossignol T, Blondin B, Dequin S, Camarasa C (2009): New insights into $\gamma$-aminobutyric acid catabolism: Evidence for $\gamma$-hydroxybutyric acid and polyhydroxybutyrate synthesis in Saccharomyces cerevisiae. Appl. Environ. Microbiol. 75, 4231-4239 https://doi.org/10.1128/AEM.00051-09

Barnett GR, Seyfzadeh M, Davis RH (1988): Putrescine and spermidine control degradation and synthesis of ornithine decarboxylase in Neurospora crassa. J. Biol. Chem. 263, 10005-10008

Bönnighausen J, Gebhard D, Kröger C, Hadeler B, Tumforde T, Lieberei R, Bergemann J, Schäfer W, Bormann J (2015): Disruption of the GABA shunt affects mitochondrial respiration and virulence in the cereal pathogen Fusarium graminearum. Mol. Microbiol. 98, 1115-1132

https://doi.org/10.1111/mmi.13203

Bouché N, Fait A, Bouchez D, Møller SG, Fromm H (2003): Mitochondrial succinic-semialdehyde dehydrogenase of the $\gamma$-aminobutyrate shunt is required to restrict levels of reactive oxygen intermediates in plants. Proc. Natl. Acad. Sci. USA 100, 6843-6848 https://doi.org/10.1073/pnas.1037532100

Bradford MM (1976): A rapid and sensitive method for the quantitation of microgram quantities of protein utilizing the principle of protein-dye binding. Anal. Biochem. 7, 248-254 https://doi.org/10.1016/0003-2697(76)90527-3

Cao J, Barbosa JM, Singh NK, Locy RD(2013): GABA shunt mediates thermotolerance in Saccharomyces cerevisiae by reducing reactive oxygen production. Yeast 30, 129-144 https://doi.org/10.1002/yea.2948

Chen D, Shao Q, Yin L, Younis A, Zheng B (2019): Polyamine function in plants: metabolism, regulation on development, and roles in abiotic stress responses. Front. Plant Sci. 9, 1945 https://doi.org/10.3389/fpls.2018.01945

Chevrot R, Rosen R, Haudecoeur E, Cirou A, Shelp BJ, Ron E, Faure D (2006): GABA controls the level of quorum-sensing signal in Agrobacterium tumefaciens. Proc. Natl. Acad. Sci. USA 103, 7460-7464 https://doi.org/10.1073/pnas.0600313103

Christensen RL, Schmit JC (1980): Regulation and glutamic acid decarboxylase during Neurospora crassa conidial germination. J. Bacteriol. 144, 983-990 https://doi.org/10.1128/JB.144.3.983-990.1980

Davis RH, Ristow JL (1989): Uptake, intracellular binding, and excretion of polyamines during growth of Neurospora crassa. Arch. Biochem. Biophys. 271, 315-322

https://doi.org/10.1016/0003-9861(89)90281-6

Ebbole DJ (1998): Carbon catabolite repression of gene expression and conidiation in Neurospora crassa. Fungal Genet. Biol. 25, 15-21 https://doi.org/10.1006/fgbi.1998.1088

Fait A, Fromm H, Walter D, Galili G, Fernie AR (2008): Highway or byway: the metabolic role of the GABA shunt in plants. Trends Plant. Sci. 13, 14-19 https://doi.org/10.1016/j.tplants.2007.10.005

Feehily C, Karatzas KA (2013): Role of glutamate metabolism in bacterial responses towards acid and other stresses. J. Appl. Microbiol. 114, 11-24 https://doi.org/10.1111/j.1365-2672.2012.05434.x

Goffeau A, Barrell BG, Bussey, Davis RW, Dujon B, Feldmann H, Galibert F, Hoheisel JD, Jacq C, Johnston M et al. (1996): Life with 6000 genes. Science 274, 545-567 https://doi.org/10.1126/science.274.5287.546

Guignard R, Grange F, Turian G (1984): Microcycle conidiation induced by partial nitrogen deprivation in Neurospora crassa. Can. J. Microbiol. 30, 1210-1215 https://doi.org/10.1139/m84-192

Guignard R., Brody S (1983): Conidium formation and germination in Neurospora crassa: Glutamic acid metabolism. Exp. Mycol. 7, 133-140 https://doi.org/10.1016/0147-5975(83)90056-7

Guo M, Chen Y, Du Y, Dong Y, Guo W, Zhai S, Zhang H, Dong S, Zhang Z, Wang Y, et al. (2011): The bZIP transcription factor MoAP1 mediates the oxidative stress response and is critical for pathogenicity of the rice blast fungus Magnaporthe oryzae. PLoS Pathog. 7, e1001302 https://doi.org/10.1371/journal.ppat.1001302

Hampe CS, Mitoma H, Manto M (2018): GABA and glutamate: Their transmitter role in the CNS and pancreatic islets. In: 
GABA And Glutamate. New Developments In Neurotransmission Research (Ed. J. Samardzic), IntechOpen Limited, London, UK

https://doi.org/10.5772/intechopen.70958

Hao R, Schmit JC (1993): Cloning of the gene for glutamate decarboxylase and its expression during conidiation in Neurospora crassa. Biochem. J. 293, 735-738

https://doi.org/10.1042/bj2930735

Hao R, Schmit JC (1991): Purification and characterization of glutamate decarboxylase from Neurospora crassa conidia. J. Biol. Chem. 266, 5135-5139

Hutchings H, Stahmann KP, Roels S, Espeso EA, Timberlake WE, Arst HN Jr, Tilburn J (1999): The multiply-regulated gabA gene encoding the GABA permease of Aspergillus nidulans: a score of exons. Mol. Microbiol. 32, 557-568 https://doi.org/10.1046/j.1365-2958.1999.01371.x

Kumar S, Punekar NS (1997): The metabolism of 4-aminobutyrate (GABA) in fungi. Mycol. Res. 101, 403-409 https://doi.org/10.1017/S0953756296002742

Kuo HC, Hui S, Choi J, Asiegbu FO, Valkonen JP, Lee YH (2014): Secret lifestyles of Neurospora crassa. Sci. Rep. 4, 5135 https://doi.org/10.1038/srep05135

Masuo S, Terabayashi Y, Shimizu M, Fujii T, Kitazume T, Takaya N (2010): Global gene expression analysis of Aspergillus nidulans reveals metabolic shift and transcription suppression under hypoxia. Mol. Genet. Genomics 284, 415-424 https://doi.org/10.1007/s00438-010-0576-x

McCluskey K (2003): The Fungal Genetics Stock Center: from molds to molecules. Adv. Appl. Microbiol. 5, 245-262 https://doi.org/10.1016/S0065-2164(03)01010-4

McCluskey K, Wiest A, Plamann M (2010): The Fungal Genetics Stock Center: a repository for 50 years of fungal genetics research. J. Biosci. 35, 119-126 https://doi.org/10.1007/s12038-010-0014-6

Mead O, Thynne E, Winterberg B, Solomon PS(2013): Characterising the role of GABA and its metabolism in the wheat pathogen Stagonospora nodorum. PLoS One 8, e78368 https://doi.org/10.1371/journal.pone.0078368

Meena M, Prasad V, Zehra, Gupta VK, Upadhyay RS (2015): Mannitol metabolism during pathogenic fungal-host interactions under stressed conditions. Front. Microbiol. 6, 1019 https://doi.org/10.3389/fmicb.2015.01019

Müller B, Russo VEA (1989): Nitrogen starvation or glucose limitation induces conidiation in constantly shaken liquid cultures of Neurospora crassa. Fungal Genet. Rep. 36, 58-60 https://doi.org/10.4148/1941-4765.1508

Nelson MA, Metzenberg RL (1992): Sexual development genes of Neurospora crassa. Genetics 132, 149-162

Nižnanský L, Kryštofová S, Vargovič P, Kaliňák M, Šimkovič M, Varečka L' (2013): Glutamic acid decarboxylase gene disruption reveals signalling pathway(s) governing complex morphogenic and metabolic events in Trichoderma atroviride. Antonie Van Leeuwenhoek 104, 793-807 https://doi.org/10.1007/s10482-013-9989-y

Nižnanský L', Kryštofová S, Varečka L' (2016): Disruption of GABA shunt affects Trichoderma atroviride response to nutritional and environmental stimuli. Acta. Chim. Slov. 9, 109-113 https://doi.org/10.1515/acs-2016-0019
Peñalva MA, Tilburn J, Bignell E. Arst HN Jr (2008): Ambient pH gene regulation in fungi: making connections. Trends Microbiol. 16, 291-300 https://doi.org/10.1016/j.tim.2008.03.006

Pokorný R, Vargovič P, Hölker U, Janssen M, Bend J, Hudecová D, Varečka L' (2005): Developmental changes in Trichoderma viride enzymes abundant in conidia and the light-induced conidiation signalling pathway. J. Basic Microbiol. 45, 219-229

https://doi.org/10.1002/jobm.200410354

Priya M, Sharma L, Kaur R, Bindumadhava H, Nair RM, Siddique KHM, Nayyar H (2019): GABA ( $\gamma$-aminobutyric acid), as a thermo-protectant, to improve the reproductive function of heat-stressed mungbean plants. Sci. Rep. 9, 7788 https://doi.org/10.1038/s41598-019-44163-w

Ramesh SA, Kamran M, Sullivan, Chirkova L, Okamoto M, Degryse F, McLaughlin, M, Gilliham M, Tyerman SD (2018): Aluminum-activated malate transporters can facilitate GABA transport. Plant Cell 30, 1147-1164 https://doi.org/10.1105/tpc.17.00864

Ravasz D, Kacso G, Fodor V, Horvath K, Adam-Vizi V, Chinopoulos C (2017): Catabolism of GABA, succinic semialdehyde or gammahydroxybutyrate through the GABA shunt impair mitochondrial substrate-level phosphorylation. Neurochem. Int. 109, 41-53 https://doi.org/10.1016/j.neuint.2017.03.008

Saude EJ, Slupsky CM, Sykes BD (2006): Optimization of NMR analysis of biological fluids for quantitative accuracy. Metabolomics 2, 113-123 https://doi.org/10.1007/s11306-006-0023-5

Schmit JC, Brody S (1975): Neurospora crassa conidial germination: role of endogenous amino acid pools. J. Bacteriol. 124, 232-242 https://doi.org/10.1128/JB.124.1.232-242.1975

Seifikalhor M, Aliniaeifard S, Hassani B, Niknam V, Lastochkina O (2019): Diverse role of $\gamma$-aminobutyric acid in dynamic plant cell responses. Plant. Cell Rep. 38, 847-867 https://doi.org/10.1007/s00299-019-02396-Z

Shelp BJ, Bozzo GG, Trobacher CP, Zarei A, Deyman KL, Brikis CJ (2012): Hypothesis/review: contribution of putrescine to 4-aminobutyrate (GABA) production in response to abiotic stress. Plant Sci. 193-194, 130-135

https://doi.org/10.1016/j.plantsci.2012.06.001

Strigáčová J, Chovanec P, Liptaj T, Hudecová D, Turský T, Šimkovič M, Varečka L (2001): Glutamate decarboxylase activity in Trichoderma viride conidia and developing mycelia. Arch. Microbiol. 175, 32-40 https://doi.org/10.1007/s002030000235

Šimkovič M, Lakatoš, B, Tsuji FI, Muto S, Varecka L (2001): The effect of azalomycin $\mathrm{F}$ on Ca2+ homeostasis in Trichoderma viride and Saccharomyces cerevisiae. Gen. Physiol. Biophys. 20, 131-144

Tiedje KE, Stevens K, Barnes S, Weaver DF (2010): $\beta$-Alanine as a small molecule neurotransmitter. Neurochem. Int. 57, $177-188$ https://doi.org/10.1016/j.neuint.2010.06.001

Tisdale HD (1967): Preparation and properties of succiniccytochrome c reductase (complex II-III). Methods Enzymol. 10, 213-215 https://doi.org/10.1016/0076-6879(67)10042-6 
Valdés-Santiago L, Cervantes-Chávez JA, León-Ramírez CG, RuizHerrera J (2012): Polyamine metabolism in fungi with emphasis on phytopathogenic species. J. Amino Acids 2012, 837932 https://doi.org/10.1155/2012/837932

Vogel HJ (1956): A convenient growth medium for Neurospora crassa (N medium). Microbial Genet. Bull. 13, 42-43

Vogel HJ (1964): Distribution of lysine pathways among fungi: evolutionary implications. Am. Nat. 98, 435-446 https://doi.org/10.1086/282338

Westergaard M, Mitchell HK (1947): Neurospora V. A synthetic medium favoring sexual reproduction. Am. J. Bot. 34, 573577

https://doi.org/10.1002/j.1537-2197.1947.tb13032.x

Received: December 23, 2019

Final version accepted: February 14, 2020 